\title{
Modeling Heavy/Medium-Duty Fuel Consumption Based on Drive Cycle Properties
}

\section{Lijuan Wang, Adam Duran, Jeffrey Gonder, and Kenneth Kelly}

National Renewable Energy Laboratory

CITATION: Wang, L., Duran, A., Gonder, J., and Kelly, K., "Modeling Heavy/Medium-Duty Fuel Consumption Based on Drive Cycle Properties," SAE Technical Paper 2015-01-2812, 2015, doi:10.4271/2015-01-2812.

Copyright (C) 2015 SAE International

\begin{abstract}
This paper presents multiple methods for predicting heavy/mediumduty vehicle fuel consumption based on driving cycle information. A polynomial model, a black box artificial neural net model, a polynomial neural network model, and a multivariate adaptive regression splines (MARS) model were developed and verified using data collected from chassis testing performed on a parcel delivery diesel truck operating over the Heavy Heavy-Duty Diesel Truck (HHDDT), City Suburban Heavy Vehicle Cycle (CSHVC), New York Composite Cycle (NYCC), and hydraulic hybrid vehicle (HHV) drive cycles. Each model was trained using one of four drive cycles as a training cycle and the other three as testing cycles. By comparing the training and testing results, a representative training cycle was chosen and used to further tune each method. HHDDT as the training cycle gave the best predictive results, because HHDDT contains a variety of drive characteristics, such as high speed, acceleration, idling, and deceleration. Among the four model approaches, MARS gave the best predictive performance, with an average percent error of $-1.84 \%$ over the four chassis dynamometer drive cycles. To further evaluate the accuracy of the predictive models, the approaches were applied to real-world data. MARS outperformed the other three approaches, providing an average percent error of $-2.2 \%$ over four real-world road segments. The MARS model performance was then compared to powertrain modeling results over HHDDT, CSHVC, NYCC, and HHV drive cycles using NREL's Future Automotive Systems Technology Simulator (FASTSim). The results indicated that the MARS method achieved comparable predictive performance with FASTSim.
\end{abstract}

\section{Introduction}

In response to demands from end users and regulators, heavy- and medium-duty (HD/MD) vehicle and engine manufacturers face pressure to develop products that maximize fuel efficiency and minimize vehicle fuel consumption (FC) and greenhouse gas (GHG) emissions. It is valuable to develop a methodology for predicting HD/ MD vehicle FC during unknown driving cycles based on the vehicle's measured FC from other cycles and the properties of these cycles as FC is highly dependent on duty cycles. Various researchers have developed simulation models and tools to predict FC over different usage patterns. Vehicle powertrain models like the Future Automotive System Technology Simulator (FASTSim) [1] and Autonomie [2] have been used for predicting fuel economy for various types of vehicles. These models provide accurate results, but require detailed specifications, which may not be readily available. Prior studies have investigated FC and emissions from different driving cycles. Taylor et al. []ㅡ argued that the nature of an engine or chassis dynamometer test cycle might affect FC and emissions through its inherent load history or transient operation content. Ericsson [4] investigated the impact of a large range of cycle properties on a vehicle's FC and emissions. Clark et al. [5] examined the ability to predict the emissions over a cycle based upon the vehicle's measured emissions from other cycles and properties of these cycles. The objective of this study was to develop a methodology for predicting HD/MD vehicle FC over an "unseen" driving cycle, based on second-by-second fuel rates from test cycles and the properties of those cycles.

\section{Methodology}

The main assumption in this modeling approach is that, for a given vehicle, FC depends on known driving cycle properties.

\section{Road Load Equation}

Vehicle FC depends on engine power demand, which can be calculated from the road load equation (Equation 1):

$$
P_{R L}=P_{a u x}+\frac{m V(d V / d t)+0.5 C_{d} \rho A V^{3}+\mu m g V \cos (\theta)+m g V \sin (\theta)}{\eta}
$$

Equation 1

where $m$ is vehicle mass $(\mathrm{kg}), V$ is vehicle speed $(\mathrm{m} / \mathrm{s}), C_{d}$ is coefficient of aerodynamic drag, $\rho$ is air density $\left(\mathrm{kg} / \mathrm{m}^{3}\right), \mu$ is the coefficient of rolling resistance, $g$ is acceleration due to gravity $\left(\mathrm{m} / \mathrm{sec}^{2}\right)$, and $\theta$ is grade angle. $P_{R L}$ denotes total engine power demanded to propel the vehicle, $P_{\text {aux }}$ denotes auxiliary load including engine cooling fan, $m V(d V / d t)$ denotes vehicle inertia, $0.5 C_{d} \rho A V^{3}$ denotes aerodynamic drag, $\mu m g V \cos (\theta)$ denotes rolling 
resistance, $m g V \sin (\theta)$ denotes grade effect, and $\eta$ represents the vehicle powertrain efficiency. The mathematical equation of the road grade was calculated as the vertical rise $(+)$ or fall $(-)$ in meters $(\mathrm{m})$ for every $100 \mathrm{~m}$.

The road load equation tells us that, under constant vehicle weight, the FC is determined by driving cycle properties (vehicle speed, acceleration, and road grade).

\section{Identification of Metrics}

The derivation of the final models involved experimentation with numerous polynomial combinations of speed and acceleration levels. Specifically, linear, quadratic, cubic, and quartic terms of speed and acceleration were investigated. The final regression models included a combination of linear, quadratic, and cubic speed and acceleration and their combination terms because it gave the least number of terms with a relatively good fit to the National Renewable Energy Laboratory's (NREL's) Renewable Fuels and Lubricants (ReFUEL) Laboratory test data.

\section{Driving Cycle Used}

In this study, several cycles that cover a wide range of driving conditions were used. The Heavy Heavy-Duty Diesel Truck (HHDDT) schedule was originally created for the E55/59 study that represented real-world truck activity in California $[\underline{6}, \underline{7}, \underline{8}]$. The HHDDT schedule included four modes, namely idle, creep, transient, and cruise modes. The creation of HHDDT has been discussed previously $[\underline{6}, \underline{7}, \underline{8}]$. Each cycle has unique idle time, average speed, stop times, acceleration, and deceleration. The creep mode has an average speed of less than two miles per hour, with a distance of 0.12 miles [] $]$. The transient mode has a higher average speed of 15.34 miles per hour, with a distance of 2.8 miles. The transient mode is the typical stop-and-go behavior for a HD truck in an urban area [ $\underline{8}]$. The cruise mode stands for freeway travel. HHDDT_s represents both the high-speed cruise that characterizes similar highway behavior that occurs in congested urban areas and south-north travel along Highway 99 and Interstate 55 []]. The City Suburban Heavy Vehicle Cycle (CSHVC) is a chassis dynamometer test for HD vehicles developed by West Virginia University [9]. The basic parameters of CSHVC include a 1,700-second duration, a 6.68 mile distance, a 14.15-mph average speed, and a 43.84-mph maximum speed [9]. The New York Composite Cycle (NYCC) is a chassis dynamometer test for HD vehicles representative of actual driving patterns in New York City [10]. The characteristics of NYCC include a 1,029-second duration, an 8.85-mph average speed, a 36.0-mph maximum speed, and a 2.52 miles distance [10]. The Hydraulic Hybrid Vehicle (HHV) drive cycle is an NREL custom cycle, which includes aggressive driving behavior representative of real-world parcel delivery vehicle driving behavior in Baltimore. Chassis dynamometer testing was performed over HHDDT, CSHVC, NYCC, and HHV on three parcel delivery truck powertrains at NREL's ReFUEL Laboratory: conventional diesel, conventional gasoline and hydraulic-diesel hybrid. Time-speed traces of these cycles are shown in Figures $1, \underline{2}, \underline{3}, \underline{4}$.

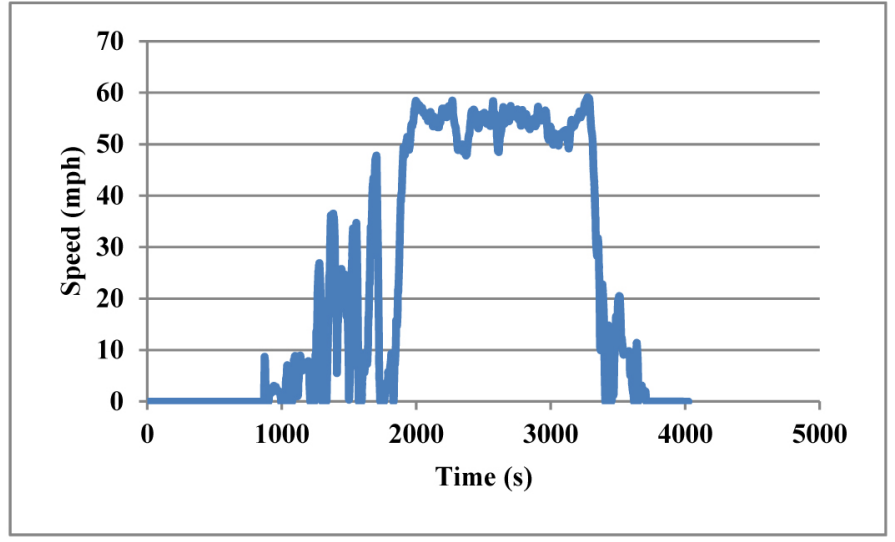

Figure 1. HHDDT speed trace.

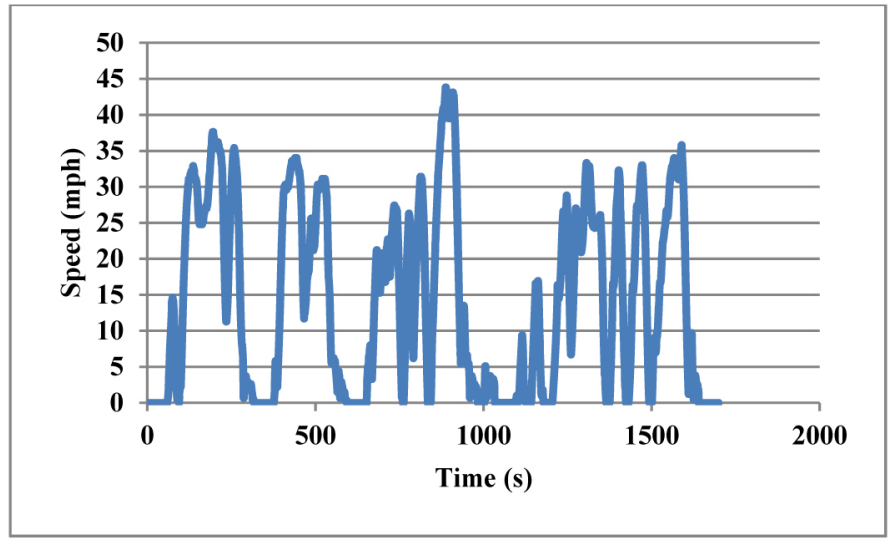

Figure 2. CSHVC speed trace.

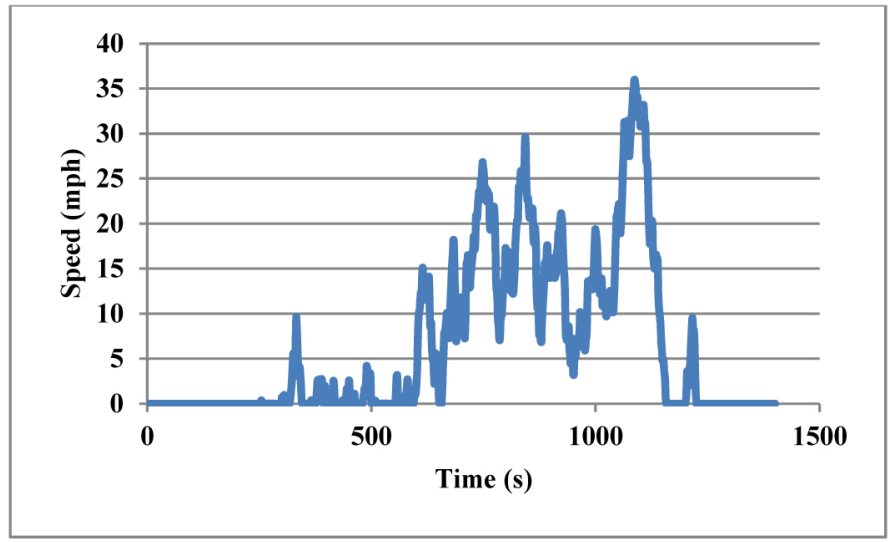

Figure 3. NYCC speed trace.

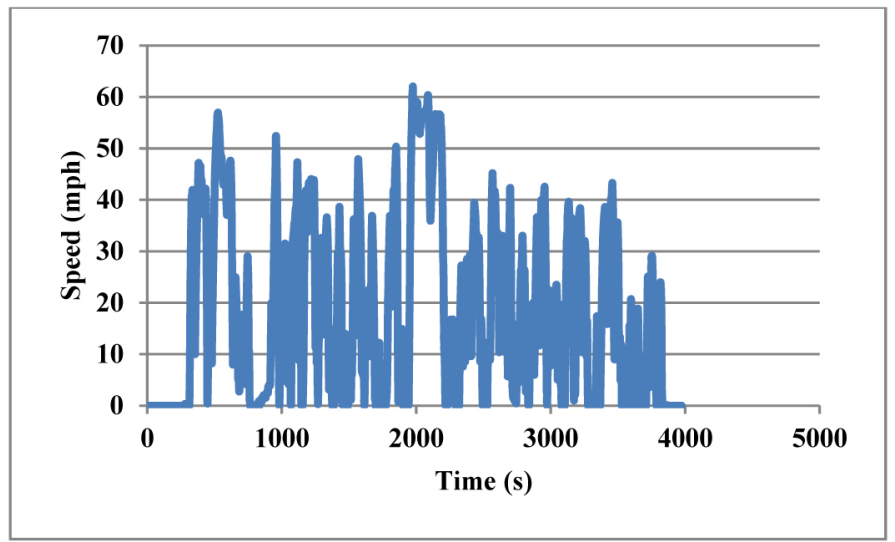

Figure 4. HHV speed trace. 


\section{Model Development}

Linear and nonlinear methods have been extensively investigated as a means to predict FC. The objective of this paper is to compare the performance of linear and nonlinear regression predictors in terms of their ability to predict FC as a function of the one fundamental input parameter-vehicle speed. A linear regression-polynomial method and three nonlinear regression methods-artificial neural network (ANN), polynomial neural network (PNN), and multivariate adaptive regression splines (MARS)-were used in this study to develop the predictive models.

\section{Polynomial Method}

The goal of the predictive method is to find a relatively simple and effective model. As stated in Equation 1, vehicle FC depends on engine power demand, which can be calculated from the road load equation. It follows from the structure of the road load equation that a logical predictive model would include a combination of vehicle speed- and acceleration-based terms. The calibration of the developed model includes selecting the most effective input parameters. The best model was obtained when the predictive FC is expressed as the real-time rate of $\mathrm{FC}$ that is a function of vehicle speed, vehicle acceleration, and combinations of these [11] (Equation 2).

$$
\begin{aligned}
& F C_{p \mathrm{re}}=a * v e h S p d^{3} * v e h A c c^{3}+b * v e h S p d^{2} * v e h A c c^{2}+c * v e h S p d^{*} v e h A c c \\
& +d * v e h S p d^{3}+e * v e h S p d^{2}+f * v e h S p d+g * v e h A c c^{2}+h * v e h A c c+i
\end{aligned}
$$

Equation 2

where $F C_{p r e}$ is the predicted FC, vehSpd is vehicle speed, and vehAcc is vehicle acceleration. Parameters a-i are constant coefficients that are estimated by performing the least squares method [12-13]. It should be noted that it can only be applied for a specific vehicle with all identified coefficients. The matrix form of the output $\mathrm{Y}$ is given by:

$$
Y=P \alpha
$$

Equation 3

where $Y$ is the dependent variable, in this case the $\mathrm{FC}$ rate:

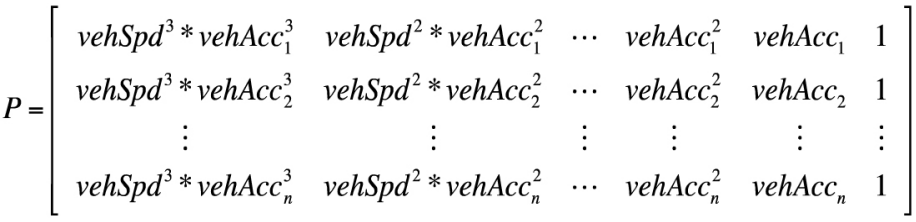

$$
\begin{aligned}
& \text { Equation } 4
\end{aligned}
$$

and $\alpha$ is the vector of the constant coefficients.

By minimizing $\|Y-P \alpha\|^{2}$, the coefficients are estimated, which is given as:

$$
\alpha=\left(P^{T} P\right)^{-1} P^{T} Y
$$

Equation 5

Once the coefficients are estimated, the predicted FC rate could be calculated by using Equation 2. The criterion that was used to evaluate the model performance between the measured and predicted $\mathrm{FC}$ is the relative error (RE), which is defined in Equation 6:

$$
R E=\frac{(\widehat{y}-y) * 100 \%}{y}
$$

Equation 6

where $y$ and $\bar{y}$ are the experimental and estimated $\mathrm{FC}$, respectively.

\section{Artificial Neural Network}

ANN modeling FC and emissions have been discussed in previous literature [14-15]. The ANN model utilized the generalized regression neural network (GRNN) [16-17] that includes a radial basis function (RBF) layer and a linear layer to achieve the emissions prediction. An RBF neuron with $R$ inputs is shown in Figure 5 . The vector distance $\|$ dist $\|$ is the dot products of the input vector $X$ and the input weight matrix. The input to the transfer function is the sum of weighted inputs and the scalar bias $b[\underline{18}]$. The input to the $i^{\text {th }}$ neuron can be expressed by Equation 7 [16].

$$
n_{i}=\sum_{i} w_{i} * \rho\left(\left\|x-x_{i}\right\|\right)
$$

Equation 7

where $\mathrm{w}_{\mathrm{i}}$ is the weight of the linear output neuron, $\mathrm{x}_{\mathrm{i}}$ is the center vector for neuron $i, \rho$ is the basis function, which is described by $\|$ dist $\|$, the distance between input vector $\mathrm{x}$ and training pattern $\mathrm{x}_{\mathrm{i}}$, and $b_{i}$ is the bias. Correspondingly, the output to the transfer function for a radial basis neuron is shown in Equation 8 [16].

$$
\operatorname{radbas}\left(n_{i}\right)=\exp \left(-n_{i}^{2}\right)=\exp \left(-\left(\sum_{i} w_{i} * \rho\left(\left\|x-x_{i}\right\|\right)\right)^{2}\right)
$$

Equation 8

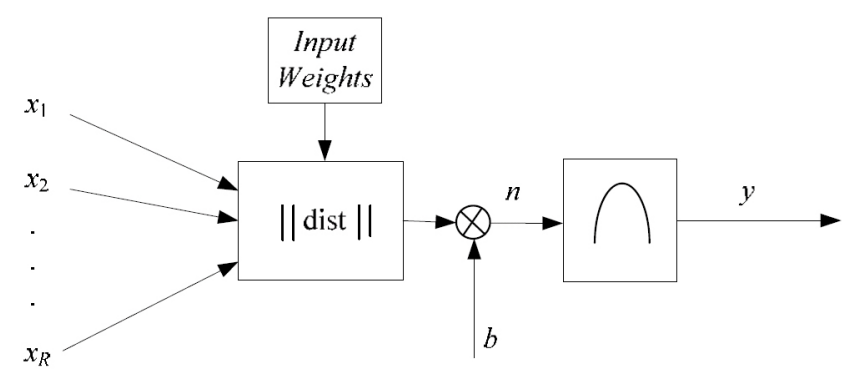

Figure 5. The RBF neuron architecture.

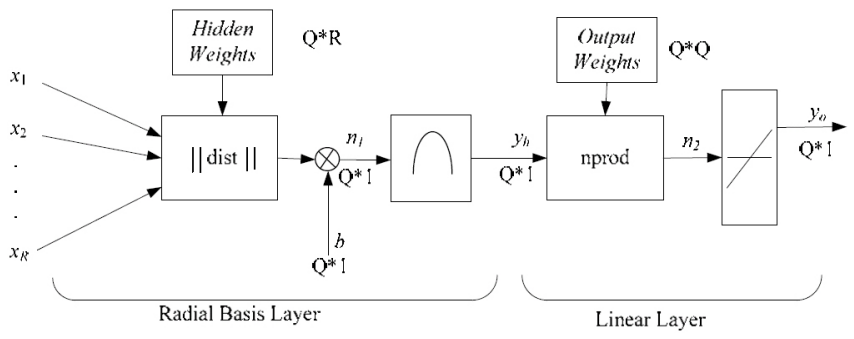

Figure 6. The GRNN architecture [18].

In MATLAB, the syntax of GRNN is given by equation 9[18]:

$$
\text { net }=\text { newgrnn }(P, T, \text { spread })
$$


where $P$ is input vector, $T$ is target vectors, and spread is the spread of RBF. All the input and target vectors were normalized before the training work. Consequently, the prediction data were also normalized and need to be de-normalized back. The normalization in this study was defined by equation 10 :

$$
y=x_{i} / x_{\max }
$$

Equation 10

where $y$ is the normalized inputs and targets, which all fall in the interval $[-1,1]$. The value of spread was varied during model development, with 0.2 providing the best match with target values.

\section{Polynomial Neural Network}

The PNN algorithm is developed based on the Group Method of Data Handling (GMDH) [19] and utilizes a class of polynomial functions. The algorithm builds the network layer by layer using training data while the structure and number of layers of the network are controlled by either the regularity criterion [20] or Akaike's information criterion [21]. The analysis and design of PNN have been discussed previously [22]. The algorithm's parameters include the maximum number of inputs of each neuron, degree of polynomials in the neurons, and the number of neurons in a layer. The PNN toolbox used in this study was downloaded from ARESLab [르]

\section{Multivariate Adaptive Regression Splines}

MARS is an adaptive piece-wise regression approach that builds a response function in terms of nonlinear component functions and their products. It has the ability to model complex and highdimensional data dependencies and has been used for diverse

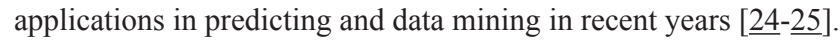
Additional information on the use of MARS is to predict engine emissions illustrated by Chen [26].

The MARS constructs models using a special set of spline functions called hinge functions or "hockey stick" functions [27]. The hinge functions are expressed in equation 11:

$$
\max (0, x-c) \quad \text { or } \quad \max (0, c-x)
$$

Equation 11

where $c$ is the knot of the basis function.

MARS uses the combination of basis functions to build the model, shown in Equation 12:

$$
\hat{f}_{i}(x)=\sum_{i=1}^{n} c_{i} B_{i}(x)
$$

Equation 12

where $B_{i}(x)$ is the $i$ th basis function, which is either a constant or a hinge function from Equation 11 or a product of two or more hinge functions.

MARS builds a model in two steps: forward selection and backward deletion. MARS starts with selecting a pair of basis functions that fit the model best at the current stage and then repeatedly adds basis function in pairs to the model. To prevent the final model from being overfitted, the backward model prunes the model. It removes the least effective term at each step until it finds the best fitted model.

\section{Results and Discussion}

The data used to evaluate the predictive models were collected from conventional diesel parcel delivery truck chassis dynamometer testing on the HHDDT, CSHVC, NYCC, and HHV driving cycles (no grades on the chassis dynamometer data).

\section{Linear (Polynomial) Method Results}

Among the four chassis dynamometer test cycles, each cycle was considered as a training cycle and the other three as testing cycles. Equation 2 was applied to build the model. By comparing the training and testing results, a representative training cycle can be chosen. Table 1 shows the summary of training and testing performance for FC. Measured Ave. FR is defined as measured average fuel rate, and Predicted Ave. FR is defined as predicted average fuel rate. The results indicate that HHDDT as a training cycle gives the best performance. This is likely because the HHDDT contains a variety of driving characteristics, such as idling, accelerating, cruise, and decelerating. In the following nonlinear study (ANN, PNN and MARS), the HHDDT was chosen as the training cycle and the other three were used as the testing cycles.

\begin{tabular}{|c|c|c|c|c|c|}
\hline \multicolumn{2}{|l|}{ Cycle } & $\begin{array}{l}\text { HHDDT } \\
\text { Testing }\end{array}$ & $\begin{array}{l}\text { CSHVC } \\
\text { Testing }\end{array}$ & $\begin{array}{l}\text { NYCC } \\
\text { Testing }\end{array}$ & HHV \\
\hline \multirow{3}{*}{$\begin{array}{l}\text { HHDDT } \\
\text { Training }\end{array}$} & $\begin{array}{l}\text { Measured. } \\
\text { Ave. FR } \\
(\mathrm{L} / \mathrm{h})\end{array}$ & 8.53 & 5.67 & 4.51 & 8.47 \\
\hline & $\begin{array}{l}\text { Predicted. } \\
\text { Ave. FR } \\
\text { (L/h) }\end{array}$ & 8.53 & 5.71 & 4.34 & 7.55 \\
\hline & RE (\%) & 0.00 & -0.74 & 3.75 & 10.89 \\
\hline \multirow{3}{*}{$\begin{array}{l}\text { CSHVC } \\
\text { Training }\end{array}$} & $\begin{array}{l}\text { Measured. } \\
\text { Ave. FR } \\
\text { (L/h) }\end{array}$ & 8.53 & 5.67 & 4.51 & 8.47 \\
\hline & $\begin{array}{l}\text { Predicted. } \\
\text { Ave. FR } \\
(\mathrm{L} / \mathrm{h})\end{array}$ & 6.66 & 5.67 & 4.20 & 6.60 \\
\hline & RE (\%) & 21.83 & 0.00 & 6.73 & 22.06 \\
\hline \multirow{3}{*}{$\begin{array}{l}\text { NYCC } \\
\text { Training }\end{array}$} & $\begin{array}{l}\text { Measured. } \\
\text { Ave. FR } \\
\text { (L/h) }\end{array}$ & 8.53 & 5.67 & 4.51 & 8.47 \\
\hline & $\begin{array}{l}\text { Predicted. } \\
\text { Ave. FR } \\
(\mathrm{L} / \mathrm{h})\end{array}$ & 2.58 & 5.72 & 4.51 & 5.40 \\
\hline & RE (\%) & 69.75 & -0.91 & 0.00 & 36.29 \\
\hline \multirow{3}{*}{$\begin{array}{l}\text { HHV } \\
\text { Training }\end{array}$} & $\begin{array}{l}\text { Measured. } \\
\text { Ave. FR } \\
(\mathrm{L} / \mathrm{h})\end{array}$ & 8.53 & 5.67 & 4.51 & 8.47 \\
\hline & $\begin{array}{l}\text { Predicted. } \\
\text { Ave. FR } \\
(\mathrm{L} / \mathrm{h})\end{array}$ & 10.29 & 6.56 & 4.78 & 8.47 \\
\hline & RE (\%) & -20.64 & -15.75 & -6.16 & 0.00 \\
\hline
\end{tabular}

Table 1. Predictive results of chassis dynamometer data using the polynomial method. 


\section{Nonlinear Methods Results}

Table 2 summarizes the results of estimation using the ANN, PNN, and MARS approaches. Among these three nonlinear approaches, MARS outperforms ANN and PNN predictive performance with an average percent error of $-1.84 \%$ ( $-5.78 \%$ to $4.79 \%$ range).

Table 2. Predictive results of chassis dynamometer data using ANN, PNN and MARS approaches.

\begin{tabular}{|c|c|c|c|c|c|}
\hline \multicolumn{2}{|c|}{ Approach \Cycle } & HHDDT & CSHVC & NYCC & $\mathrm{HHV}$ \\
\hline \multirow[t]{3}{*}{ ANN } & $\begin{array}{l}\text { Measured. } \\
\text { Ave. FR } \\
(\mathrm{L} / \mathrm{h})\end{array}$ & 8.53 & 5.67 & 4.51 & 8.47 \\
\hline & $\begin{array}{l}\text { Predicted. } \\
\text { Ave. FR } \\
(\mathrm{L} / \mathrm{h})\end{array}$ & 8.40 & 6.35 & 4.37 & 8.39 \\
\hline & RE (\%) & 1.43 & -11.96 & 3.01 & 0.99 \\
\hline \multirow[t]{3}{*}{ PNN } & $\begin{array}{l}\text { Measured. } \\
\text { Ave. FR } \\
(\mathrm{L} / \mathrm{h})\end{array}$ & 8.53 & 5.67 & 4.51 & 8.47 \\
\hline & $\begin{array}{l}\text { Predicted. } \\
\text { Ave. FR } \\
(\mathrm{L} / \mathrm{h})\end{array}$ & 8.53 & 6.06 & 4.20 & 7.74 \\
\hline & $\mathrm{RE}(\%)$ & 0.00 & 6.89 & -6.67 & -8.65 \\
\hline \multirow[t]{3}{*}{ MARS } & $\begin{array}{l}\text { Measured. } \\
\text { Ave. FR } \\
(\mathrm{L} / \mathrm{h})\end{array}$ & 8.53 & 5.67 & 4.51 & 8.47 \\
\hline & $\begin{array}{l}\text { Predicted. } \\
\text { Ave. FR } \\
\text { (L/h) }\end{array}$ & 8.53 & 5.94 & 4.24 & 8.09 \\
\hline & RE (\%) & 0.00 & 4.79 & -5.79 & -4.53 \\
\hline
\end{tabular}

\section{Application to Real-World Drive Cycles}

In order to further evaluate the accuracy of the proposed FC prediction model, real world data were applied to these models. The real-world data used were collected by Penske Truck Rental Company on four different road segments. Figure 7 shows the vehicle speed trace on the four road segments, as well as the road elevation information. Segment 3 was taken as a training cycle and the other three segments as testing cycles. The predictive results of real world data using polynomial, ANN, PNN, and MARS approaches are summarized in Table 3. As was observed from the chassis dynamometer testing comparisons, MARS gave the best predictive performance with an average percent error of $-2.2 \%(-6.83 \%$ to $3.73 \%$ range). It should be noted that the grades were not included due to the lack of grade information, which might be one of the reasons why segment 4 with uphill had the largest underestimation error. The model performance should be improved if accurate grade information was accessible.
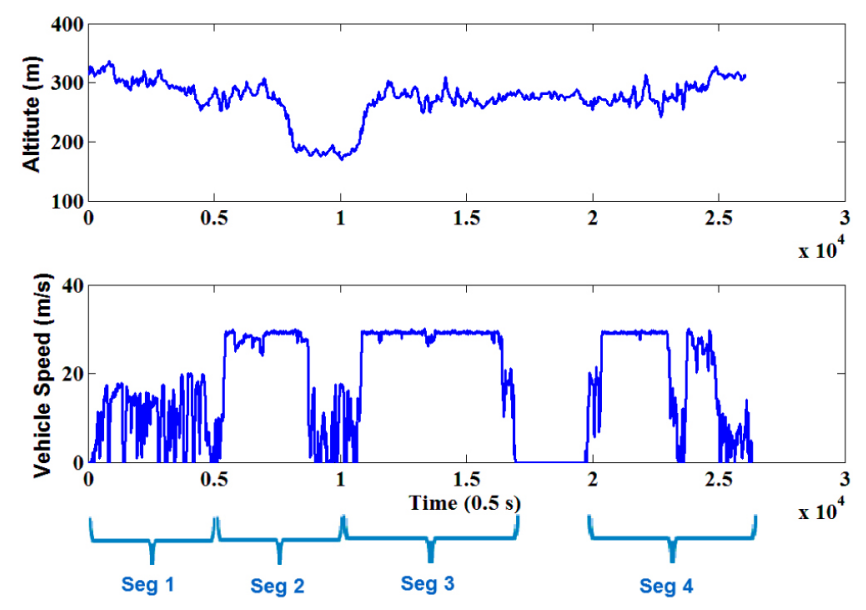

Figure 7. Real world road altitude and road speed trace.

Table 3. Predictive results of real world data using polynomial, ANN, PNN and MARS approaches.

\begin{tabular}{|c|c|c|c|c|c|}
\hline \multicolumn{2}{|c|}{ Approach $\backslash$ Segment } & \multirow{2}{*}{$\begin{array}{l}\text { Seg3 } \\
\text { Training/Testing } \\
30.11\end{array}$} & \multirow{2}{*}{$\begin{array}{l}\text { Seg1 } \\
\text { Testing } \\
13.70\end{array}$} & \multirow{2}{*}{$\begin{array}{l}\text { Seg2 } \\
\text { Testing } \\
24.79\end{array}$} & \multirow{2}{*}{$\begin{array}{l}\text { Seg4 } \\
\text { Testing } \\
26.39 \\
\end{array}$} \\
\hline Polynomial & $\begin{array}{l}\text { Measured. } \\
\text { Ave. FR } \\
(\mathrm{L} / \mathrm{h})\end{array}$ & & & & \\
\hline & $\begin{array}{l}\text { Predicted. } \\
\text { Ave. FR } \\
(\mathrm{L} / \mathrm{h})\end{array}$ & 30.11 & 14.21 & 24.84 & 23.92 \\
\hline & RE (\%) & 0.00 & 3.74 & -0.21 & -9.39 \\
\hline \multirow[t]{3}{*}{$\mathrm{ANN}$} & $\begin{array}{l}\text { Measured. } \\
\text { Ave. FR } \\
(\mathrm{L} / \mathrm{h})\end{array}$ & 30.11 & 13.70 & 24.79 & 26.39 \\
\hline & $\begin{array}{l}\text { Predicted. } \\
\text { Ave. FR } \\
\text { (L/h) }\end{array}$ & 30.11 & 15.42 & 24.01 & 26.70 \\
\hline & RE (\%) & 0.00 & -12.53 & 3.15 & -1.17 \\
\hline \multirow[t]{3}{*}{$\mathrm{PNN}$} & $\begin{array}{l}\text { Measured. } \\
\text { Ave. FR } \\
(\mathrm{L} / \mathrm{h})\end{array}$ & 30.11 & 13.70 & 24.79 & 26.39 \\
\hline & $\begin{array}{l}\text { Predicted. } \\
\text { Ave. FR } \\
(\mathrm{L} / \mathrm{h})\end{array}$ & 30.11 & 14.78 & 25.58 & 22.98 \\
\hline & RE (\%) & 0.00 & 7.88 & 3.21 & -12.91 \\
\hline \multirow[t]{3}{*}{ MARS } & $\begin{array}{l}\text { Measured. } \\
\text { Ave. FR } \\
(\mathrm{L} / \mathrm{h})\end{array}$ & 30.11 & 13.70 & 24.79 & 26.39 \\
\hline & $\begin{array}{l}\text { Predicted. } \\
\text { Ave. FR } \\
(\mathrm{L} / \mathrm{h})\end{array}$ & 30.11 & 14.21 & 23.92 & 24.59 \\
\hline & $\mathrm{RE}(\%)$ & 0.00 & 3.73 & -3.50 & -6.83 \\
\hline
\end{tabular}

\section{Comparison with FASTSim}

FASTSim is a vehicle powertrain simulation tool developed by NREL [1] that is used to evaluate the impact of technology improvements on efficiency, performance, cost, and battery life in conventional vehicles, hybrid electric vehicles, plug-in hybrid electric vehicles, and all-electric vehicles. A FASTSim conventional diesel truck model (the truck information is presented in Table 4) was developed and run 
over the HHDDT, CSHVC, NYCC, and HHV cycles, and then the results were compared with that of the MARS model, summarized in Table 5.

Table 4. Information on the conventional diesel truck model.

\begin{tabular}{|l|l|}
\hline Vehicle Type & Diesel Conventional Truck \\
\hline Vehicle Tested Weight (lb) & 15,410 \\
\hline Coefficient of Rolling Resistance & 0.0071 \\
\hline Coefficient of Aerodynamic Drag & 0.71 \\
\hline Frontal Area (m $\left.{ }^{2}\right)$ & 6 \\
\hline Rated Engine Power (HP) & 200 \\
\hline
\end{tabular}

Table 5. Predictive results of FASTSim and MARS approaches.

\begin{tabular}{|c|c|c|c|c|c|}
\hline \multicolumn{2}{|c|}{ Approach $\backslash$ Cycle } & HHDDT & CSHVC & NYCC & HHV \\
\hline \multirow{3}{*}{ FASTSim } & $\begin{array}{l}\text { Measured. } \\
\text { Ave. FR } \\
(\mathrm{L} / \mathrm{h})\end{array}$ & 8.53 & 5.67 & 4.51 & 8.47 \\
\hline & $\begin{array}{l}\text { Predicted. } \\
\text { Ave. FR } \\
(\mathrm{L} / \mathrm{h})\end{array}$ & 8.72 & 5.60 & 4.17 & 8.92 \\
\hline & RE (\%) & 2.24 & -1.27 & -7.53 & 5.34 \\
\hline \multirow{3}{*}{ MARS } & $\begin{array}{l}\text { Measured. } \\
\text { Ave. FR } \\
(\mathrm{L} / \mathrm{h})\end{array}$ & 8.53 & 5.67 & 4.51 & 8.47 \\
\hline & $\begin{array}{l}\text { Predicted. } \\
\text { Ave. FR } \\
(\mathrm{L} / \mathrm{h})\end{array}$ & 8.53 & 5.94 & 4.24 & 8.09 \\
\hline & RE (\%) & 0.00 & 4.79 & -5.79 & -4.53 \\
\hline
\end{tabular}

The comparison shows that for this situation (where FASTSim relies on generalized component efficiency maps in the absence of vehicle-specific component details) that the simplified MARS approach achieves comparable predictive results.

\section{Summary}

This paper proposed a FC predictive model taking instantaneous vehicle speed and acceleration as input variables. Four modeling algorithms-polynomial, ANN, PNN, and MARS-were developed and verified using chassis dynamometer testing on diesel parcel delivery trucks operating on HHDDT, CSHVC, NYCC, and HHV driving cycles at the ReFUEL Laboratory. MARS gave the best predictive performance with an average percent error of $-1.84 \%$ for the four chassis dynamometer test cycles. The models were also applied to real world data for further evaluation of the accuracy. MARS gave the best predictive results with an average percent error of $-2.2 \%$. Ultimately, the performance of the MARS approach was compared with that of FASTSim; the results showed that the MARS approach achieved an accuracy comparable to FASTSim.

The models presented in this paper can be incorporated with traffic simulation models. They can also be applied directly to estimate vehicle FC and emissions using recorded global positioning system, speed, and road grade information (e.g., to predict individualized fuel consumption in a fleet considering purchase of a vehicle for which such a simplified model has been developed).

\section{References}

1. National Renewable Energy Laboratory, "Future Automotive Systems Technology Simulator,” http://www.nrel.gov/ transportation/fastsim.html, accessed April 6, 2015.

2. Argonne National Laboratory, “Autonomie,” http://www. autonomie.net, accessed April 6, 2015.

3. Taylor, S.T., Clark, N.N., Gautam, M., and Wayne, W.S., "Diesel Emissions Prediction from Dissimilar Cycle Scaling," Journal of Automobile Engineering 218:341-352, 2004.

4. Ericsson, E., "Independent Driving Pattern Factors and Their Influence on Fuel-Use and Exhaust Emission Factors," Transportation Research Part D 6:325-345, 2001.

5. Clark, N.N., Wang, L., Vora, K.A., Gautam, M., Wayne, W.S., and Thompson, G., "Expressing Cycles and Their Emissions on the Basis of Properties and Results from Other Cycles," Environ. Sci. Technol.44: 5986-5992, 2010.

6. Clark, N.N., Gautam, M., Wayne, W.S., Thompson, G.J. and Lyons, D.W., "California Heavy Heavy-Duty Diesel Truck Emissions Characterization for Project E-55/E-59 Phase 1.5," CRC Project E-55/E-59 Report, August 2004.

7. U.S. Environmental Protection Agency, "Code of Federal Regulations, CFR Appendix I to Part 86 Urban Driving Dynamometer Driving Schedule, Title 40," U.S. Government Printing Office, Washington, D.C., 2005.

8. Clark, N., Gautam, M., Wayne, W., Nine, R. et al., "Creation and Evaluation of a Medium Heavy-Duty Truck Test Cycle," SAE Technical Paper 2003-01-3284, 2003, doi:10.4271/200301-3284.

9. DieselNet, "City Suburban Heavy Vehicle Cycle \& Route," https://www.dieselnet.com/standards/cycles/csc.php, accessed April 6, 2015.

10. DieselNet, "New York Composite Cycle," https://www. dieselnet.com/standards/cycles/nycomp.php, accessed April 6, 2015.

11. Clark, N., Bedick, C., Wang, L., Thompson, G. et al., "Emissions from a Legacy Diesel Engine Exercised through the ACES Engine Test Schedule," SAE Technical Paper 2008-011679, 2008, doi:10.4271/2008-01-1679.

12. Wolberg, J., "Data Analysis Using the Method of Least Squares: Extracting the Most Information from Experiments," (Springer, New York, NY, 2005).

13. Saville, D.J., and Wood, G.R., "Statistical Methods: The Geometric Approach, Third Edition,” (Springer, New York, NY, 1991).

14. Desantes, J., López, J., García, J., and Hernández, L., "Application of Neural Networks for Prediction and Optimization of Exhaust Emissions in a H.D. Diesel Engine," SAE Technical Paper 2002-01-1144, 2002, doi:10.4271/200201-1144.

15. De Lucas, A., Duran, A. Carmona M., and Lapuerta, M., "Modeling Diesel Particulate Emissions with Neural Networks," Fuel 80(4): 539-548, March 2001. 
16. Buhmann, M.D., "Radial Basis Functions: Theory and Implementations," (Cambridge University Press, Cambridge, U.K., 2003).

17. Celikoglu, H.B., "Application of Radial Basis Function and Generalized Regression Neural Networks in Non-Linear Utility Function Specification for Travel Mode Choice Modeling," Mathematical and Computer Modeling 44(7-8): 640-658, October 2006.

18. MathWorks, "Generalized Regression Neural Networks," http:// www.mathworks.com/help/nnet/ug/generalizedregressionneural-networks.html, accessed April 7, 2015.

19. Farlow, S.J., "The GMDH algorithm," in Farlow SJ, editor, "Self-organizing Methods in Modeling: GMDH Type Algorithms," (Marcel Dekker, New York, NY, 1984), 1-24.

20. Bastian, A., and Gasos, J., "Modeling Using Regularity Criterion Based Constructed Neural Networks," Computers \& Industrial Engineering 27(1-4): 441-444, September 1994.

21. MathWorks, "Akaike Information Criterion for Estimated Model,” http://www.mathworks.com/help/ident/ref/aic.html, accessed April 16, 2015.

22. Oh, S., Pedrycz, W., and Park, B., "Polynomial Neural Networks Architecture: Analysis and Design," Computers \& Electrical Engineering 29(6): 703-725, August 2003.

23. Jekabsons, G., “ARESLab: Adaptive Regression Splines Toolbox for Matlab/Octave," 2011, available at http://www. cs.rtu.lv/jekabsons.

24. Sephton, P. "Forecasting Recessions: Can We Do Better on MARS?” Fed. Res. Bank of St. Louis, 83: 39-49, 2001.

25. Das, D., Banerjee, N., and Zhang, M.Q., "Interacting Models of Cooperative Gene Regulation," Proc. Natl. Acad. Sci. USA 101: 16234-16239, 2004.

26. Chen, X., Schmid, N.A., Wang, L., and Clark N.N., "Regression-Based Oxides of Nitrogen Predictors for Three Diesel Engine Technologies," J. Air \& Waste Manage. Assoc. 60(72-90), DOI:10.3155/1047-3289.60.1.72.

27. Friedman, J.H. "Multivariate Adaptive Regression Splines," Ann. Stats. 19:1-141, 1991.

\section{Contact Information}

Lijuan Wang

Lijuan.wang@nrel.gov

\section{Acknowledgments}

This work was supported by the U.S. Department of Energy under Contract No. DE-AC36-08GO28308 with the National Renewable Energy Laboratory. Funding was provided by U.S. DOE Office of Energy Efficiency and Renewable Energy Vehicle Technologies Office.

The U.S. Government retains and the publisher, by accepting the article for publication, acknowledges that the U.S. Government retains a nonexclusive, paid-up, irrevocable, worldwide license to publish or reproduce the published form of this work, or allow others to do so, for U.S. Government purposes.

\section{Definitions/Abbreviations}

ANN - artificial neural network

CSHVC - City Suburban Heavy Vehicle Cycle

FASTSim - Future Automotive Systems Technology Simulator

FC - fuel consumption

GHG - greenhouse gas

GMDH - Group Method of Data Handling

GRNN - generalized regression neural network

HD - heavy-duty vehicle

HHDDT - Heavy Heavy-Duty Diesel Truck

HHV - hydraulic hybrid vehicle

MARS - multivariate adaptive regression splines

MD - medium-duty

NREL - National Renewable Energy Laboratory

NYCC - New York Composite Cycle

PNN - polynomial neural network

RBG - radial basis function

ReFUEL - Renewable Fuels and Lubricants

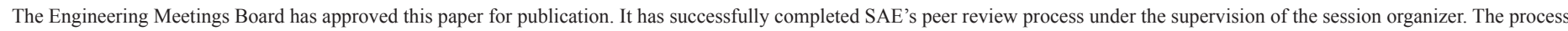
requires a minimum of three (3) reviews by industry experts.

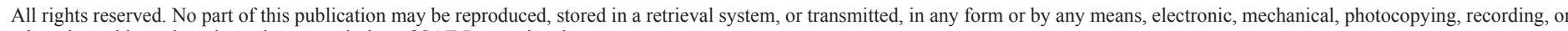
otherwise, without the prior written permission of SAE International.

Positions and opinions advanced in this paper are those of the author(s) and not necessarily those of SAE International. The author is solely responsible for the content of the paper.

ISSN 0148-7191

http://papers.sae.org/2015-01-2812 